\title{
Aberrant lipid metabolism reprogramming and immune microenvironment for gastric cancer: a literature review
}

\author{
Meng-Ying Cui ${ }^{\#} \wedge$, Xing Yi” ${ }^{\#}$, Dan-Xia Zhu, Jun Wu^ \\ Department of Oncology, The Third Affiliated Hospital of Soochow University, Changzhou, China \\ Contributions: (I) Conception and design: DX Zhu, J Wu, MY Cui; (II) Administrative support: DX Zhu, J Wu; (III) Provision of study materials \\ or patients: DX Zhu, J Wu, MY Cui; (IV) Collection and assembly of data: MY Cui, X Yi; (V) Data analysis and interpretation: MY Cui, X Yi; \\ (VI) Manuscript writing: All authors; (VII) Final approval of manuscript: All authors. \\ "These authors contributed equally to this work. \\ Correspondence to: Jun Wu; Dan-Xia Zhu. Department of Oncology, The Third Affiliated Hospital of Soochow University, 185 Juqian St., Changzhou \\ 213003, China. Email: wujun1378@163.com; zhudanxia66@163.com.
}

\begin{abstract}
Objective: We summarize the aberrant lipid metabolism disorders associated with enzyme activity and expression changes and related immune microenvironment for gastric cancer.

Background: Gastric cancer is a malignant tumor of the primary digestive system with high incidence, poor prognosis characterized by extensive metastasis and poor effect with radiotherapy and chemotherapy. One of the most important metabolic characteristics of cancer cells is lipid metabolism reprogramming to adapt to the tumor micro-environment.

Methods: The focus of research in recent years has also been on lipid metabolism disorders, particularly aberrant metabolism of fatty acids (FAs) in gastric cancer cells, as well as an upregulation of the expression and activity of key enzymes in lipid metabolism. These changes remind us of the occurrence and development of gastric cancer. These metabolic changes are not unique to cancer cells. Changes in metabolic procedures also determine the function and viability of immune cells. In the immune microenvironment of gastric cancer, the metabolic competition and interaction between cancer cells and immune cells are not very clear, while a deeper understanding of the topic is critical to targeting the differential metabolic requirements of them that comprise an immune response to cancer offers an opportunity to selectively regulate immune cell function.
\end{abstract}

Conclusions: Recent research suggests that targeting metabolism is an emerging and potentially promising treatment strategy for gastric cancer patients. We need to explore it further.

Keywords: Lipid metabolism related enzymes; targeted metabolism treatment; tumor immunosuppression microenvironment; gastric cancer

Submitted Apr 17, 2021. Accepted for publication Jul 05, 2021.

doi: $10.21037 /$ tcr-21-655

View this article at: https://dx.doi.org/10.21037/tcr-21-655

\section{Introduction}

Among the tumors of the digestive system, the incidence of gastric cancer has always been high. The overall survival (OS) rate of advanced gastric cancer (AGC) or recurrent, unresectable gastric cancer is very depressing. In China, the five-year survival rate is only $35.9 \%$ (1), and the mortality rate remains very high. Therefore, it is urgent for us to further explore and develop new treatments. The deficiency of nutrients in the tumor immunosuppression microenvironment may lead to metabolic competition between tumor cells and surrounding immune cells

^ ORCID: Meng-Ying Cui, 0000-0003-2929-3735; Xing Yi, 0000-0001-6505-0437; Jun Wu, 0000-0003-2929-3735. 
[including tumor-associated macrophages (TAMs), dendritic cells (DCs), bone marrow-derived suppressors cells, and $\mathrm{T}$ lymphocytes], weakening the immune response to promote tumor proliferation, diffusion and metastasis (2). Aberrant lipid metabolism in gastric cancer is a relatively new field that has been widely concerned in recent years and targeted metabolism is a brand-new treatment strategy that can benefit patients with gastric cancer. This review aims to explore the metabolic disorders caused by aberrant lipid metabolism and related synthetase activity changes in gastric cancer cells and adjacent immune cells, which contributes to the formation of an immunosuppressive microenvironment that supports the progression of gastric cancer and explore the effects of targeted treatments with aberrant lipid metabolism related enzymes (3). We assess their potential as molecular targets for further benefiting gastric cancer patients.

We present the following article in accordance with the Narrative Review reporting checklist (available at https:// dx.doi.org/10.21037/tcr-21-655).

\section{Aberrant lipid metabolism of gastric cancer}

In normal cells, lipid synthesis, lipid droplet storage, and fatty acid oxidation (FAO) are in dynamic balance. In order to meet the demand for raw materials from the continuous expansion of cancer cells, gastric cancer cells take in more exogenous fats through CD36 receptors (4) while becoming more reliant on de novo synthesis pathways. Pyruvate, the final product of glycolysis, undergoes the tricarboxylic acid cycle to produce intermediate citric acid, which is cleaved by ATP citrate lyase (ACLY) to produce acetyl-CoA, an important raw material for the synthesis of cholesterol and fatty acids (FAs) (5). This process is finely regulated by the activation of sterol regulatory element binding protein-1 (SREBP-1), and the PI3K-AKT-mTOR signaling path, which is a unique conversion from sugar to lipid in malignant cells (6). It is worth mentioning that acetate can also be converted into citric acid by the ACSS2 enzyme and subsequently into acetyl-CoA by ACLY, which is less common pathway for acetyl-CoA synthesis (7). Acetyl$\mathrm{CoA}$ is used as a synthetic raw material in two ways. One of them is the irreversible conversion to malonyl- CoA by acetyl-CoA carboxylase (ACC). It is worth mentioning that two different types of ACC finely regulate the synthesis and decomposition of the oxidation capacity of this FA. Malonyl-CoA is continuously added to form palmitic acid after seven consecutive cycles of acyl transfer, condensation, reduction, dehydration and redox by fatty acid synthase (FASN). Of course, saturated FAs can further be elongated or desaturated by stearoyl-CoA desaturases (SCDs) to form MUFA/PUMA, eventually forming cholesterol ester and triglycerides, which are stored in lipid droplets or used for cell membrane synthesis. In the other way, acetylCoA forms cholesterol, an important raw material for cell membranes, through the mevalonate pathway (MVP) (8). In the first stage, acetyl-CoA generates acetoacetyl-CoA under the action of acetoacetyl-CoA thiolase, which is then converted into HMG-CoA by the key enzyme of hydroxymethylglutaryl-CoA (HMG-CoA synthetase). In the second stage, HMG-CoA generates mevalonate under the action of HMG-CoA reductase. Finally, through a series of chemical reactions, such as phosphorylation, decarboxylation, and dehydroxylation, isoprene and $27 \mathrm{C}$ cholesterol are synthesized (9). When cancer cell expansion requires energy, long-chain fatty acids (LCFAs) are taken into the blood and enter the mitochondria through the CPT1 rate-limiting enzyme (carnitine acyltransferase 1), they then undergo continuous dehydrogenation, water addition, dehydrogenation, and thiolysis in FAO (10). The solution eventually produces acetyl-CoA, releasing a large amount of energy and reduction equivalents, such as NADPH and FADH2, for the growth and metastasis of gastric cancer cells and alleviating self-oxidative stress damage (11).

The latest research shows that the typical characteristics of lipid metabolism in gastric cancer are increased lipid synthesis and up-regulated $\beta$-oxidation and oxidative decomposition of FAs (12). For general cancer cells, SCAP/ SREBPs can link tumorigenic signals with changes in lipid metabolism (13). After SREBPs are activated by the PI3K/AKT signaling pathway, it promotes ACLY, ACC, FASN, stearoyl-CoA desaturase 1 (SCD1) and low-density lipoprotein receptor (LDLR) expression to enhance fatty acid synthesis (FAS) and cholesterol uptake (14). Although the specific regulatory mechanism in gastric cancer patients is not fully understood, key endogenous factors such as stearoyl-coenzyme a desaturase (SCD), FASN and acetylcoenzyme a carboxylase (ACC) are indeed involved in the occurrence of gastric cancer (15). Among them, the increase of FAS expression is closely related to gastric tumor proliferation, chemotherapy resistance and poor prognosis (16). In addition to the de novo synthesis pathway unique to cancer cells, gastric cancer cells directly modify CD36 by activating the NF- $\kappa \mathrm{B}$ pathway to increase the level of O-GlcNAc acylation. CD36 up-regulates DEK 
transcription (17), and epithelial-mesenchyme mediated by GSK-3 $\beta / \beta$-catenin quality transformation promotes the migration and invasion of gastric cancer cells, which in turn promotes the high uptake of exogenous fat by gastric cancer cells, forming a vicious circle of gastric cancer metastasis (18). It is worth mentioning that plasma oxLDL is also related to lymphatic metastasis of gastric cancer, and lipoprotein-mediated cholesterol entry and steroid production are biomarkers that lead to the progression of gastric cancer (19). Specifically, the mechanism mainly involves oxLDL activating the NF- $\mathrm{KB}$ signaling pathway mediated by LOX-1, followed by the up-regulation of VEGF-C expression. And oxLDL is secreted from gastric cancer cells and ultimately promotes the rapid lymphatic metastasis of gastric cancer (20). Liver X receptors (LXRs) are also involved in lipid metabolism disorders in gastric cancer. LXR $\beta$ agonists inhibit Wnt signaling through LXR $\beta$ relocation, thereby inhibiting the proliferation of gastric cancer cells (21). However, the specific process has not been further confirmed. The expression of cholesterol glycosyltransferase (encoded by Cgt) in Helicobacter pylori is necessary for HP colonization in the stomach and the activation of T cells (22). The MVP and cholesterol homeostasis are an indispensable part of cancer cells, which are highly correlated with the growth, survival and metastatic potential of gastric cancer cells (23). In the case of Helicobacter pylori infection, cholesterol supply enhances JAK/STAT signaling and the release of antibacterial effect substances (24). Unexpectedly, this increased Th1 response would be considered a promoter of tumorigenesis (25). This idea coincides with elevated blood cholesterol levels, especially low-density lipoprotein-cholesterol (26). These patients generally have severe gastritis caused by Helicobacter pylori. Therefore, exogenous low cholesterol intake may reduce the occurrence of Helicobacter pylori infection and gastric cancer. This closely links Cgt function, cholesterol metabolism and inflammation with the occurrence and development of gastric cancer (27). The latest research shows that the oxidative phosphorylation of mitochondria in gastric cancer cells is driven by NADH provided by fatty acid $\beta$ oxidation (28). Compared with benign diseases of the stomach (such as chronic superficial gastritis), in gastric cancer tissue, FAs involved in lipid metabolism, such as hexadecenoic acid, docosahexaenoic acid, valeric acid and $\beta$-hydroxybutyric acid, were significantly increased (29). It shows that fatty acid $\beta$ oxidation is decomposed more rapidly in the tumor immune microenvironment (30). As gastric cancer progresses, the metabolism from lipids to FAs is accelerated, and ketone bodies are eventually produced to consume fat. This phenomenon may explain the weight loss of patients with AGC (31). In the future, the exploration of abnormal lipid metabolism during the occurrence of gastric cancer will provide a new light for the treatment of gastric cancer (Figure 1).

\section{Key regulatory factors in lipid metabolism pathways}

Treatment of gastric cancer mainly takes the following three approaches: (I) limiting the source of lipids; (II) blocking the utilization of lipids; (III) blocking the formation of lipid droplets. Key regulators of lipid synthesis include SREBPs, ACLY, ACC, FASN and SCD1. Their expression is significantly up-regulated in gastric cancer. This review discusses the effects of these substances in detail and discuss their potential as molecular targets in the treatment of gastric cancer.

\section{Limiting the source of lipids}

ACLY: ATP citrate lyase is a key enzyme in the de novo FAS pathway that converts citric acid into oxaloacetate and cytosolic acetyl-CoA (32). It is also the first step in guiding glucose-derived metabolites towards the fate of lipid biosynthesis. In addition, ACLY is also regulated by the oncogenic PI3K/Akt signal pathway and is an attractive target for the treatment of malignant tumors (33). When citric acid is transported into the cytoplasm, it is cleaved by ACLY into the essential biosynthetic precursor of FA and MVP-acetyl-CoA. Acetyl-CoA is an important part of FA, cholesterol and isoprenoid biosynthesis (6). Recent studies have shown that ACLY may be used as a biomarker to predict the progression and prognosis of gastric cancer. ACLY is highly expressed in patients with gastric adenocarcinoma, which is not only conducive to the growth of cancer cells but also closely related to lymph node metastasis and advanced disease stage (34).

ACC: acetyl-CoA carboxylase can carboxylate acetylCoA to malonyl-CoA, and this is the rate-limiting step that catalyzes the de novo synthesis of FAs (35). There are two subtypes of ACC. Because their positions in sub-cells differ, they also perform different metabolic functions. Cytoplasmic ACC1 is present in adipogenic tissues, ACC1 is locally cytosolic and produces malonyl-CoA for the synthesis of FAs. On the contrary, ACC2 bound to the outer 


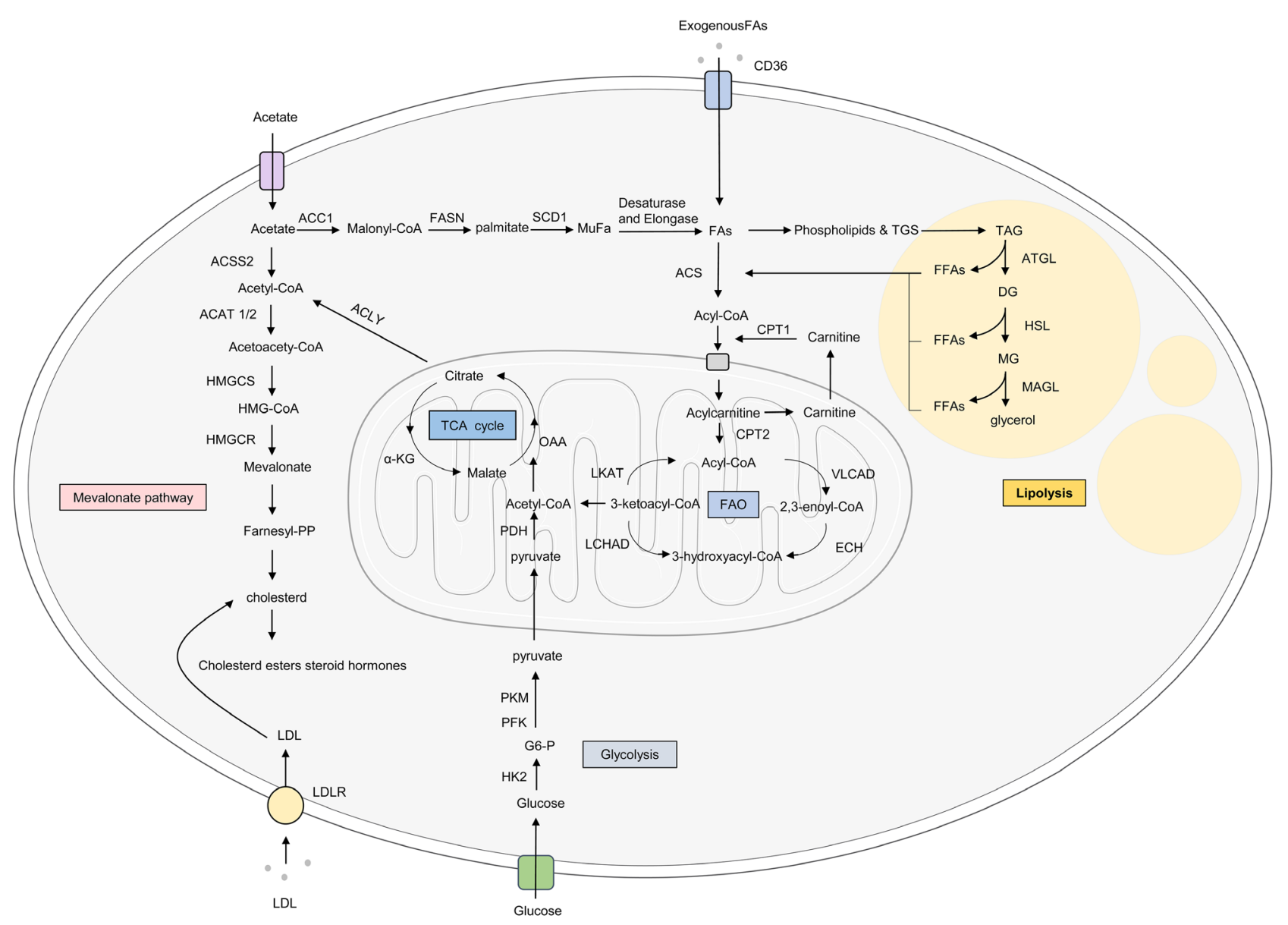

Figure 1 Overview of fatty acid metabolism in gastric cancer cells. ACLY, ATP-citrate lyase; ACC, acetyl-CoA carboxylase; FASN, fatty-acid synthase; SCD, stearoyl-CoA desaturase; ACSS, Acetyl-CoA synthetase; ACS, acyl-CoA synthetases; CPT1, carnitine palmitoyl transferase 1; CPT2, carnitine palmitoyl transferase 2; ACAT1/2, acetyl-CoA acetyltransferase 1/2; ATGL, fatty triglyceride lipase; HSL, hormonesensitive lipase; MAGL, monoacylglycerol lipase. TGs/CE, triglycerides/cholesteryl esters; TAG, triacylglycerol; DAG, diacylglycerol; MAG, monoacylglycerol; FFA, free fatty acid; FAs, fatty acids; FAO, fatty acid oxidation; TCA cycle, tricarboxylic acid cycle; NADH, nicotinamide adenine dinucleotide; $\alpha$-KG, $\alpha$-ketoglutarate; OAA, oxaloacetate; Glucose 6-P, glucose 6-phosphate; LDLR, low-density lipoprotein receptor; GLUT, glucose transporter; HK2, hexokinase 2; PFK, phosphofructokinase; PKM, pyruvate kinase M; PDH, pyruvate dehydrogenase; MUFAs, monounsaturated fatty acids; FAS, fatty acid synthesis; HMG-CoA, hydroxy-3-methyl-glutaryl-CoA; HMGCR, hydroxy-3-methyl-glutaryl-CoAreductase; HMGCS, 3-Hydroxy-3-Methylglutaryl Co-enzyme A Synthase ; LDs, lipid droplets.

mitochondrial membrane is mainly present in lipid-oxidized tissues, which inhibiting CPT1 from $\beta$-oxidation of FAs (36). In short, ACC1 is involved in lipid synthesis, and ACC2 inhibits lipid degradation (32). For gastric cancer patients, especially those without lymph node metastasis, low expression or deletion of $\mathrm{pACC}$ is related to the progression of tumor stage, the presence of lymph node metastasis and poor prognosis. High expression of pACC predicts a better prognosis in patients with primary gastric cancer resection.
These findings further suggest that ACC/pACC may be a potential molecular target for the prevention and treatment of gastric cancer (37).

FASN: fatty acid synthase is a multi-enzyme protein complex that catalyzes the de novo biosynthesis of saturated FAs (38). Palmitic acid (16:0) was produced by adding one acetyl-CoA molecule and seven malonyl-CoA molecules. Subsequently, it was elongated by ELOVL6 (extension of very long chain FA protein 6) and then desaturated by SCDs 
(stearoyl coenzyme a desaturase) (39). The high expression of FASN in gastric cancer tissues and that its expression negatively correlated with the expression of PTEN can both explain the progression and peritoneal dissemination of gastric cancer (40). At the same time, FASN is more frequently expressed in gastric cancer, adenoma, regenerating epithelium and intestinal metaplasia tissues. According to reports that the expression of FASN plays an important role in the formation of gastric precancerous lesions, but it has no obvious influence on the progression of gastric cancer (41). The activation of SREBP-1 through the SDF-1a/CXCR4 axis may promote the up-regulation of FASN expression in a large number of cancers, including gastric cancer (42). Experiments have shown that FASN is significantly related to the depth of gastric cancer invasion, lymph node metastasis and total stage. Through further research, FASN may become a clinical prognostic indicator of human gastric cancer and a therapeutic target for controlling the peritoneal spread of gastric cancer (43).

SCDs: stearoyl-CoA desaturases are endoplasmic reticulum (ER) enzymes that catalyze the conversion of saturated FAs into $\delta$-9 monounsaturated fatty acids (MUFAs) (6). Oleic acid and palmitoleic acid, the products of the SCD enzyme, are the most abundant unsaturated FAs and important precursors for the formation of complex lipids (including phospholipids, triglycerides, cholesterol esters, and diacylglycerols) (44). Therefore, the activity of this enzyme may have a wide range of effects on the growth and proliferation, energy storage and signal transduction in the process of malignant tumors (45). Tumor cells regulate SCDs by stimulating SREBPs and change the synthesis of important lipids (such as FAs, diglycerides, cholesterol and phospholipids). This phenomenon fully demonstrates that these lipids are key drivers of tumor transformation and cancer progression. For example, oleic acid omental adipocytes activate the PI3K-Akt signaling pathway, which enhances the invasiveness of gastric cancer cells (46). Increased levels of MUFAs can rescue the migration and invasion defects of colorectal cancer cells that cause SCD1 deficiency (44).

Acetyl-CoA synthetase 2 (ACSS2): it is generally believed that tumor cells can take part of acetic acid (47) and convert it into acetyl-CoA after being catalyzed by ACSS2 to form the necessary raw materials for FA and cholesterol biosynthesis (48). In fact, cancer cells mainly rely on acetate as a carbon source to synthesize FAs under hypoxic conditions. In common malignant tumors such as breast cancer, liver cancer, and lung cancer, knocking out ACSS2 can inhibit the proliferation and growth of cancer cell lines (7).
When glucose supply is limited, cancer cells mainly rely on acetate as a carbon source for FAS under conditions of extreme glucose deficiency (49). Surprisingly, the lack of ACSS2 expression accounts for a large proportion of gastric cancer $(62.6 \%)$, and this is more common in two types of poorly differentiated gastric adenocarcinoma or signet ring cell carcinoma. The latest research (48) shows that ACSS2, which is normally expressed in gastric epithelial tissue, may be inhibited during tumorigenesis or tumor progression. However, we do not know why gastric cancer cells shut down this pathway and did not use ACSS2 to provide intermediate products. Research by Huang et al. (50) demonstrated that the lack of SIRT3 expression (down-regulation of ACSS2) has a certain correlation with the rapid progression and poor prognosis of gastric cancer patients. In other words, ACSS2 deficiency is a prognostic marker for predicting worse progression-free survival and OS in patients with gastric cancer. To our surprise, the expression of mitochondrialderived acyl-CoA synthase superfamily 3 (ACSS3) is significantly higher in gastric cancer tissues, and ACSS3 knockout can inhibit the growth and invasion of gastric cancer cells. This finding not only reveals the importance of ACSS3 as an important prognostic biomarker for gastric cancer but also suggests targeting ACSS3 as a potentially promising treatment approach (19).

HMGCR: hydroxy-3-methyl-glutaryl-CoAreductase, most studies reported that the up-regulation of FA and cholesterol-related enzymes is necessary for the progression of highly proliferative malignant tumors (51). The most important step in the MVP is the conversion of HMG-CoA to mevalonate by the rate-limiting enzyme HMGCR of MVP. Among the MVP targeted drugs, statins, as competitive inhibitors of HMGCR, can not only inhibit the proliferation and angiogenesis of gastric cancer cells, but also inhibit their migration and invasion. The latest research (52) proves that lovastatin + docetaxel provides a new anti-cancer strategy for sensitive or drugresistant gastric malignancies, and it is expected to become an adjuvant therapy for patients with gastric cancer. Many experimental studies found that the progression of gastric cancer is mainly mediated through the uptake of exogenous cholesterol through the lipoprotein/receptor (L/R) pathway (19). In contrast, it has nothing to do with the de novo synthesis of nascent cholesterol (cholesterol mediated by HMGCR) (53). Although the key enzymes for cholesterol production do not affect the progression of GCa, the enzymes involved in the low MVP (such as MVK, LSS, DHCR14A1, SC4MOL, HSD17B7, SC5D) 
promote the 5-year OS rate of GCa patients at different levels (19) HMGCR is up-regulated in gastric cancer tissues and activates the Hedgehog/Gli1 signaling pathway, suggesting that statins combined with cyclopamine, a small molecule inhibitor of Hedgehog signaling, may be effective in the treatment of gastric cancer (51).

\section{Blocking the use of lipids}

CPT1: LCFAs entering the mitochondria for oxidation require carnitine palmitoyltransferase (CPT) as a key enzyme to assist mitochondria entry for FAO (11). The FAO enhancement process is one of the important metabolic characteristics of tumor cells. It connects the tricarboxylic acid cycle to produce redox equivalents such as ATP, NADPH, NADH and FADH2 (54), which can generate energy and regulate the redox homeostasis in cells, reducing the metabolism of cancer cells stress and enhancing antioxidant capacity. As the important role of FAO in cancer being further understood, CPTI has once again become a focus of research. The high expression of CPT1C induced by hypoxia is closely related to the poor prognosis of gastric cancer and can promote the proliferation of gastric cancer cells (10). In gastric cancer patients with greater omentum metastasis, FAO is enhanced by the up-regulation of CD36 and CPT1B expression (55), which greatly increases the chance of metastasis. In addition, the inhibition of FA catabolism can enhance the efficacy of oxaliplatin-based chemotherapy in gastrointestinal tumors, mainly through the inhibition of CPT-mediated FA catabolism combined with conventional chemotherapy, which is a promising treatment strategy (56).

\section{Blocking the decomposition of lipid droplets}

Lipid droplets are dynamic organelles that can decompose and release free FAs by the action of fatty triglyceride lipase (ATGL), hormone sensitive lipase (HSL) and monoacylglycerol lipase (MAGL) when gastric cancer cells need them (57). The latest research shows that the lipolysis of FA mediated by MAGL can promote the spread, metastasis, and growth of cancer cells (58). Targeting and inhibiting the breakdown of lipid droplets to release free FAs is thus an effective treatment strategy to cut off the nutrient supply and manage the stress of cancer cells (59). Lipid droplets, as the consolidation and regulation center of the lipid acquisition pathway of cancer cells, are attractive targets for cancer cell stress management (60).

\section{The role of PI3K/AKT/mTOR pathways in lipid metabolism in gastric cancer}

In the immune microenvironment of gastric cancer, the phosphatidylinositol 3-kinase (PI3K)-serine-threonine kinase (Akt)-mammalian target of rapamycin (mTOR) pathway is abnormally activated, which stimulates the abnormal proliferation of malignant tumors. This pathway is not only an important factor in the growth, metabolism, metastasis, and resistance of gastric cancer cells to radiotherapy and chemotherapy, but also a key target for tumor immunotherapy today (61). Lipid metabolism reprogramming is an emerging marker of malignant tumors (62). The PI3K-AKT-mTOR pathway mainly regulates the abnormal de novo synthetic fats and exogenous lipids intake in gastric cancer by controlling SREBPs (63). In gastric cancer tissue, the abnormal activation of SREBP1c promotes the expression of CD36 (encoding transmembrane FA uptake transporter), ACLY, ACC, FASN and SCD1 to enhance the formation of new cell membranes and lipid rafts. SREBP-2 uses LDLR as a carrier to take up cholesterol in the blood, and to activate the key enzyme in the cholesterol synthesis pathway [3-hydroxyglutaryl-coenzyme A (HMG$\mathrm{CoA})$ reductase] to enhance cholesterol synthesis (64). The uptake and enhancement of gastric cancer cells will eventually fulfill the need of continuously expanding gastric cancer cells for lipids (65). Then, inhibitors of the PI3K/Akt/mTOR signaling pathway play an important role in gastric cancer treatment. First of all, the prospects, safety and efficacy of mTOR inhibitors (such as sirolimus and everolimus) have been demonstrated in clinical trials. Secondly, MK-2206, a highly selective isotope Akt inhibitor, inhibited the growth of gastric cancer cells by enhancing MAPK-dependent autophagy when combined with everolimus to inhibit the $\mathrm{PI} 3 \mathrm{~K} / \mathrm{Akt} / \mathrm{mTOR}$ signaling pathway (66). The enhanced anti-tumor response of this combination therapy makes it a suitable and promising strategy for the second-line treatment of gastric cancer and esophageal cancer patients receiving chemotherapy or targeted therapy (67). Additionally, the therapeutic value of PP242, a potent and selective small molecule double mTORC1/2 inhibitor (68) has been demonstrated through its double inhibition of lapamycininduced mTORC1/2 kinase activity in gastric cancer cells in vitro through both direct anti-proliferation and indirect interference with angiogenesis. Finally, the new dual PI3K/ mTOR inhibitor BEZ235 can reduce the regulation of $\mathrm{PI} 3 \mathrm{~K} / \mathrm{mTOR}$ signaling protein alone or in combination with paclitaxel, achieving cell proliferation inhibition in vitro and 
in vivo. These results provide strong evidence that BEZ235 can enhance the anti-tumor response of paclitaxel for treating gastric cancer (69).

\section{Reprogramming of lipid metabolism in gastric cancer micro-environment}

In recent years, immunotherapy has been in a major role in tumor treatment. Therefore, the metabolic connection between cancer cells and the surrounding immune cells has also become a critical topic to be comprehensively understood. Activated immune cells and cancer cells share similar metabolic pathways in certain aspects, but they also compete for basic nutrients in the tumor microenvironment (TME). Besides, research has found that the metabolic pathways of immune cells are closely related to their immune functions. Therefore, connecting tumor internal metabolism and immunotherapy by understanding the key metabolic differences between gastric cancer cells and activated immune cells in the TME may be a specific target of research for enhancing tumor immunotherapy.

\section{The effect of lipid metabolism on TAMs in gastric cancer}

TAMs play a vital role in the TME of a variety of solid malignant tumors. There are two sub-types of TAMs with completely different functions. M1-like TAMs are activated by pro-inflammatory signals (such as interferon- $\gamma$ and lipopolysaccharide) and contribute to anabolic metabolism, including glycolysis and pentose phosphate pathway (PPP) and FAS, as well as activating $\mathrm{T}$ cell adaptation immune response to exert anti-tumor effects. M1-like macrophages have different degrees of infiltration in four different clinical types of gastric cancer. They not only play an important role in the blockade effect of programmed cell death protein 1 (PD-1)/PD-L1, but are also related to better prognosis of gastric cancer patients (70). In combination with the TNM staging system, M1-like macrophages can provide improved risk stratification assessment and a more comprehensive evaluation of the prognosis of gastric cancer patients. The latest research shows that the number of infiltrating M2 macrophages and total TAMs may be poor prognostic factors for patients with gastric cancer, while M1 macrophage infiltration is associated with better survival rate (71). At the same time, the phenotypic changes of macrophages are closely linked to cancer cell lipid metabolism reprogramming and it guides macrophages to exert completely different immune functions (72). The research of $\mathrm{Wu}$ et al. took oleate as an example to prove that the metabolism of LCFAs (especially unsaturated FAs) controls the immunosuppressive phenotype of TAMs (73). The free FAs exiting the lipid droplet contribute to the polarization of M2 type TAMs. Zhang et al. suggested that the reprogramming of FA metabolism in cancer cells may affect the function of TAMs by changing the amount of FAs in the TME (74). Therefore, regulating the source of FAs in cancer cells or targeting FA metabolism in TAMs and cancer cells can become a novel strategy of solid malignant tumor treatment. It is not yet fully understood how M2-like TAMs use FAs from the microenvironment and $\beta$-oxidation. However, when the $\beta$-oxidation of cancer cells increases and results in an increase in the secretion of FAs, it will activate the surrounding M2 type TAM polarization. If the uptake and oxidation of FAs in cancer cells are artificially blocked, the transformation of M2 in TAM into M1 can be induced in a therapeutic manner, which activates the adaptive immunity of $\mathrm{T}$ cells and changes the immunosuppressive microenvironment, thereby inhibiting the development and metastasis of tumors. Therefore, further research into the lipid metabolism of macrophages in gastric cancer plays a critical role in the development of novel treatments for tumors (12).

\section{The effect of lipid metabolism on $\mathbf{T}$ cells in gastric cancer}

$\mathrm{T}$ cells are mainly divided into two categories: CD8+ T cells that mediate the direct lysis of target cells, and CD4+ T cells that secrete cytokines and form and coordinate immune responses. The latter can be further divided into helper $\mathrm{T}$ cells (Th1, Th2, Th17 type), regulatory T cells (Treg). In the immune microenvironment of gastric cancer, $\mathrm{T}$ cells infiltrating around the tumor compete with gastric cancer cells for the three major nutrients (carbohydrates, lipids and proteins), and immune cells are thus deficient in nutrition. However, changes in the metabolic status of $\mathrm{T}$ cells affect their activation, proliferation and differentiation status. The interaction between cancer cells and neighboring immune cells supports tumor growth and progression by changing the lipid metabolism (75). For activated $T$ cells to carry out anti-tumor effects, lipid synthesis including cholesterol and FAs is urgently needed to provide raw materials for their continuous clonal expansion and secretion of multiple cytokines. At the same time, PI3K and mTOR finely regulate this process by mediating the SREBP transcription 
factor and LXR transcription factor. Among them, SREBP1c mainly controls the synthesis of FAs, SREBP2 mainly controls the synthesis of cholesterol. CD8+ T cells lacking SREBP activity are expected to have insufficient cholesterol and FAS. Phospholipids and cholesterol, the raw materials in cell membranes, are not enough to generate sufficient antigen stimulation, destroying the tumor-killing function of infiltrating CD8+ cytotoxic T cells. Acyl-CoA acyltransferase 1 (ACAT1) converts free cholesterol into cholesterol esters and stores them in lipid droplets (76). The ACAT1 inhibitor avasimibe not only inhibits the esterification of cholesterol in tumor cells, thereby reducing tumor cell proliferation and metastasis, but also enhances the anti-tumor activity of various cytotoxic $\mathrm{T}$ lymphocytes and the secretion of cytotoxic factors, which has potential therapeutic significance. Tregs also play a vital role in suppressing effector cell proliferation, immune response, immune evasion and tolerance in the immune microenvironment of gastric cancer. It is closely related to the progression and poor outcome of gastric cancer and the expression of Foxp3 and the TNM stage of gastric cancer patients. Compared to most effector $\mathrm{T}$ cells that preferentially use aerobic glycolysis as the main energy source, CD8+ T cell memory cells, CD4+ Treg cells, and marrow-derived suppressor cells (MDSCs)-mediated T cells mainly rely on $\beta$-oxidation of FAs to produce energy. Therefore, the oxidation of FAs is crucial to the formation of the immunosuppressive microenvironment. Tumor-derived cholesterol can increase ER stress, leading to the expression of PD-1 in T cells, which are depleted in the tumor immune microenvironment (77). PD-1 is an immunosuppressive molecule, the signaling pathway of which promotes the FAO of endogenous FAs by up-regulating the expression of CPT1A and ATGL and lipolysis. As a result, the pathway improves the use efficiency of FAO, reduces the function of effector $\mathrm{T}$ cells by changing the metabolic program, and prevents $\mathrm{T}$ cell differentiation, thereby achieving the purpose of down-regulating the immune response and promoting tumor escape and self-tolerance (78). Lipid metabolism is also very important for maintaining the balance between Teffs and Tregs. In activated Teffs, the main source of intake of exogenous FAs and endogenous FAS, these two ways provide raw materials for the proliferation and division of $\mathrm{T}$ cells. At the end of the immune response, the surviving $\mathrm{T}$ cells will resume lipid oxidation, metabolize and become memory $\mathrm{T}$ cells to respond to the next tumor attack (79). Tregs mainly rely on FAO, which can inhibit the activation of Teffs by increasing the expression of PD-1, promoting CPT1A and inhibiting the secretion of IFN- $\gamma$. It also promotes the generation of Treg cells by activating the MAPK signaling pathway (80). Yuan et al. (81) proved that through the production of COX-2/PGE (2) in the microenvironment of gastric cancer, tumors can increase the expression of Foxp3 in tumor-infiltrated Treg cells and inhibit the proliferation of autologous CD4 (+) CD25 (-) T cells. Therefore, the use of COX inhibitors and PGE (2) receptor-specific antagonists can reverse the immunosuppression of $\mathrm{T}$ cells, which is a new breakthrough in the treatment of human gastric cancer. The increased expression of Foxp3 in tumor-infiltrated Treg cells inhibits $\mathrm{T}$ cell proliferation and promotes gastric cancer progression in a COX-2 dependent manner (82). However, the relationship between tumor infiltrating $\mathrm{T}$ cells and gastric cancer is still unclear. The latest research shows that the increase in the number of Treg cells is positively correlated with TNM staging and the degree of differentiation of gastric malignant tumors (77). However, improving the immunosuppression of Treg cells remains a big challenge for the limited gastric cancer treatments available. In addition, because the high levels of IL- 6 and TGF- $\beta$ in the microenvironment of gastric cancer continuously stimulate the production of TH17 and Treg, Treg promotes immune evasion through the production of TGF- $\beta$, and TH17 produces pro-inflammatory effects through the secretion of IL-17. Such synergistic actions promote the occurrence and progression of gastric cancer. The newly synthesized FAs under the action of ACC1 will affect the differentiation of Treg and helper T cells (Th17) (83), indicating the importance of newly synthesized FAs in determining the development of different $\mathrm{T}$ cell lines (84). However, it is unclear whether lipids affect the imbalance of Th17/Treg cells (85). Therefore, a better understanding of the impact of lipid metabolism reprogramming on $\mathrm{T}$ cells in gastric cancer is needed to facilitate the development of novel and effective gastric cancer immunotherapy (80). Therefore, the lipid metabolism reprogramming of $\mathrm{T}$ cells around gastric malignant tumors is an emerging research field.

\section{The effect of lipid metabolism on other immune cells in gastric cancer}

DCs are not only the strongest antigen presenting cells, but also the key cells that regulate activation and tolerance in the adaptive immune response (86). The differentiation and activation states of DCs determine their immune regulatory functions. In the immune microenvironment of gastric cancer, DCs as sentinels continuously uptake, process and 
present antigens (Ag), secreting cytokines and effectively activating the initial $\mathrm{T}$ cells to produce the key highefficiency immunity answer. The latest experiments show that necessary and coordinated lipid metabolism changes occur when DC is activated, and the dysregulation of lipid metabolism in gastric cancer is related to the reduced capacity for immune stimulation (87). When Msr1 was upregulated in DCs exposed to tumors, they continuously absorbed surrounding cholesterol and FAs, which then accumulated in the cytoplasm (88). The results suggest that the ability of DCs to cross-express and present Ag was reduced, eventually leading to insufficient antigen-specific activation of CD8+ T cells (89). The number of DCs is related to the staging and prognosis of gastric malignant tumors. Postoperative adjuvant immunotherapy is also beneficial to the survival of gastric cancer patients with reduced tumor DC infiltration.

Bone MDSCs are considered a class of powerful contributors in immunosuppressive TMEs. Research has shown that a high percentage of MDSC is an independent predictor of the poor prognosis of patients with AGC (90). In patients with AGC receiving cisplatin-based chemotherapy, the greater the number of MDSCs before chemotherapy, the shorter the progression-free survival and OS. This shows that MDSCs play a key role in the immune dysfunction in gastric cancer. Similarly, the higher the proportion of MDSCs around gastric cancer tissue, the more malignant a patient's tumor is, and this is often associated with the poor outcome of antiPD-1 treatment. MDSC may potentially be underlying the resistance to anti-PD-1 therapy. Combining PD-1 blockade with MDSC-targeting treatment may be a promising strategy for overcoming the resistance to immune blockade in gastric cancer patients (91). Prolonged exposure to unsaturated FAs (oleate and linoleate) triggers the inhibitory capacity of MDSC cell lines, which is often linked to a poor prognosis (92). It is well-known that if CD36 deficiency can also downregulate lipid metabolism, it prevents the immunosuppressive function of MDSCs (93). Tumor MDSCs absorb FAs from the TME, and a large amount of lipid accumulation can be seen in the cytoplasm. Lipid-overloaded MDSCs also enhance the immunosuppressive effect on CD8+ T cells, thereby promoting tumor progression. In gastric cancer, nivolumab, the third-line treatment for unresectable advanced or recurrent patients, works by depleting MDSCs and has been investigated in phase I clinical trials. The bone marrow-derived suppressor cells (T-MDSCs) infiltrated in the TME, in addition to preventing $\mathrm{T}$ cell function and enhancing the effect of tumor chemotherapy and radiotherapy, support the expansion of Treg to further strengthen this highly immunosuppressive microenvironment. At the same time, FAO is activated. CPT1A is up-regulated, and the oxygen consumption rate (OCR) is greatly increased (94). On the contrary, inhibiting FAO blocks the tolerance and immunosuppressive mechanisms of T-MDSCs, activates T cells to function, and improves cancer treatment effects (95). More importantly, inhibiting FAO enhanced the anti-tumor effects of lowdose chemotherapy and adoptive cell therapy (ACT). In addition, the induction of high expression of CPT1C in the TME is closely related to the proliferation of gastric cancer cells, as well as the poor prognosis of gastric cancer patients. Therefore, targeting FAO can be a novel strategy for antitumor treatment by targeting the lipid metabolism of gastric cancer immune cells (Figure 2).

\section{Conclusions}

Research on lipid metabolism in gastric cancer is lackingspecific mechanism and treatment-related targets are still at the pre-clinical stage and not fully validated in clinical trials. However, preliminary outcomes from research to date support its potential as the next innovative treatment after radiotherapy, chemotherapy and targeted therapy. In the immune microenvironment of gastric cancer, immune cells and gastric cancer cells compete for the required nutrients and undergo a series of metabolic changes. These differential metabolisms provide a new direction of research for targeted metabolism. Successful translation of these research findings to effective treatment demands further understanding of the differences between the metabolism of immune cells and cancer cells, as well as the identification of key lipid enzyme targets and specific mechanisms to be exploited for developing therapeutic inhibitors, and put them into practice for clinical trials and research, this will be another major breakthrough after 


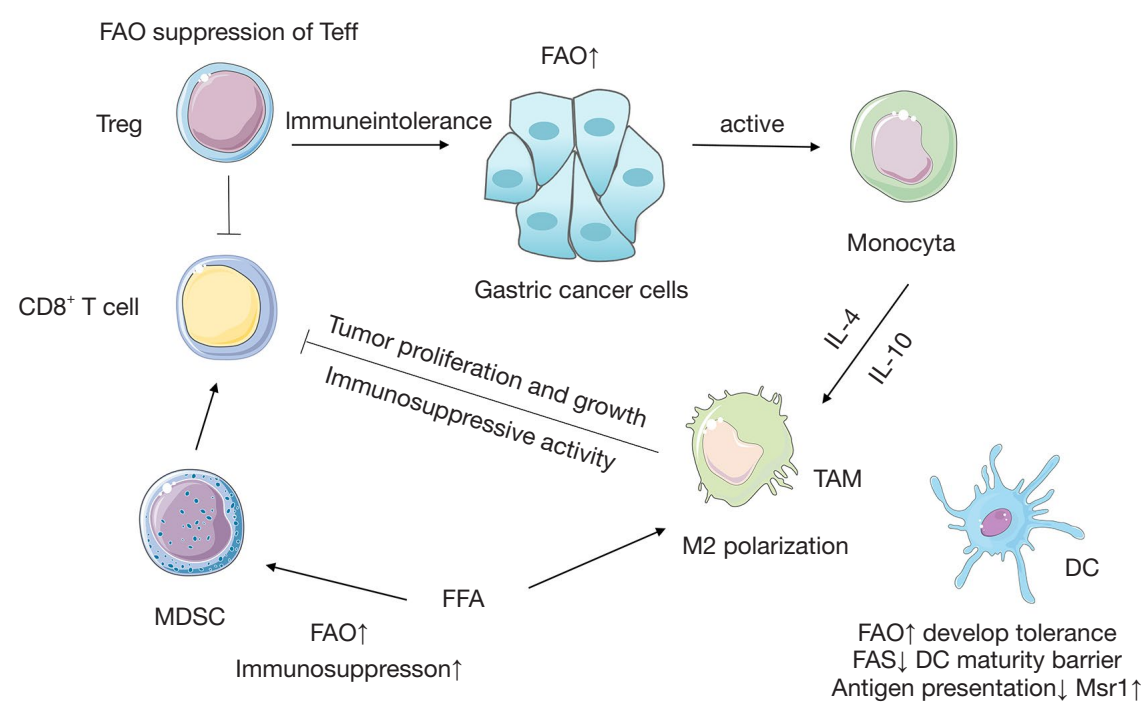

Figure 2 Gastric cancer cells and immune cells interact in the immune microenvironment. MDSC, marrow-derived suppressor cells; DCs, dendritic cells; FAO, fatty acid oxidation; FAS, fatty acid synthesis; TAMs, tumor-associated macrophages; FFA, free fatty acid; TME, tumor microenvironment; Tregs, regulatory T cells. Figures were produced using Servier Medical Art (http://www.servier.com/).

immunotherapy.

\section{Acknowledgments}

Funding: This study was supported by grants from National Natural Science Foundation of China (nos. 81770212, to Dr. Dan-Xia Zhu).

\section{Footnote}

Reporting Checklist: The authors have completed the Narrative Review reporting checklist. Available at https:// dx.doi.org/10.21037/tcr-21-655

Conflicts of Interest: All authors have completed the ICMJE uniform disclosure form (available at https://dx.doi. org/10.21037/tcr-21-655). All authors report this study was supported by grants from National Natural Science Foundation of China (nos. 81770212, to Dr. Dan-Xia Zhu) and the full text was polished by the professional native speaker Dan-Xia Zhu. The authors have no other conflicts of interest to declare.

Ethical Statement: The authors are accountable for all aspects of the work in ensuring that questions related to the accuracy or integrity of any part of the work are appropriately investigated and resolved.
Open Access Statement: This is an Open Access article distributed in accordance with the Creative Commons Attribution-NonCommercial-NoDerivs 4.0 International License (CC BY-NC-ND 4.0), which permits the noncommercial replication and distribution of the article with the strict proviso that no changes or edits are made and the original work is properly cited (including links to both the formal publication through the relevant DOI and the license). See: https://creativecommons.org/licenses/by-nc-nd/4.0/.

\section{References}

1. Glimelius B, Ekström K, Hoffman K, et al. Randomized comparison between chemotherapy plus best supportive care with best supportive care in advanced gastric cancer. Ann Oncol 1997;8:163-8.

2. Leone RD, Powell JD. Metabolism of immune cells in cancer. Nat Rev Cancer 2020;20:516-31.

3. Hu B, Lin JZ, Yang XB, et al. Aberrant lipid metabolism in hepatocellular carcinoma cells as well as immune microenvironment: A review. Cell Prolif 2020;53:e12772.

4. Pan J, Fan Z, Wang Z, et al. CD36 mediates palmitate acid-induced metastasis of gastric cancer via AKT/GSK3 $\beta / \beta$-catenin pathway. J Exp Clin Cancer Res 2019;38:52.

5. Luengo A, Gui DY, Vander Heiden MG. Targeting Metabolism for Cancer Therapy. Cell Chem Biol 2017;24:1161-80. 
6. Cheng C, Geng F, Cheng X, et al. Lipid metabolism reprogramming and its potential targets in cancer. Cancer Commun (Lond) 2018;38:27.

7. Schug ZT, Peck B, Jones DT, et al. Acetyl-CoA synthetase 2 promotes acetate utilization and maintains cancer cell growth under metabolic stress. Cancer Cell 2015;27:57-71.

8. Matsushita Y, Nakagawa H, Koike K. Lipid Metabolism in Oncology: Why It Matters, How to Research, and How to Treat. Cancers (Basel) 2021;13:474.

9. Huang B, Song BL, Xu C. Cholesterol metabolism in cancer: mechanisms and therapeutic opportunities. Nat Metab 2020;2:132-41.

10. Chen T, Wu G, Hu H, et al. Enhanced fatty acid oxidation mediated by CPT1C promotes gastric cancer progression. J Gastrointest Oncol 2020;11:695-707.

11. Qu Q, Zeng F, Liu X, et al. Fatty acid oxidation and carnitine palmitoyltransferase I: emerging therapeutic targets in cancer. Cell Death Dis 2016;7:e2226.

12. Xiao S, Zhou L. Gastric cancer: Metabolic and metabolomics perspectives (Review). Int J Oncol 2017;51:5-17.

13. Cheng C, Ru P, Geng F, et al. Glucose-Mediated $\mathrm{N}$-glycosylation of SCAP Is Essential for SREBP-1 Activation and Tumor Growth. Cancer Cell 2015;28:569-81.

14. Guo D, Bell EH, Mischel P, et al. Targeting SREBP-1driven lipid metabolism to treat cancer. Curr Pharm Des 2014;20:2619-26.

15. Nannini G, Meoni G, Amedei A, et al. Metabolomics profile in gastrointestinal cancers: Update and future perspectives. World J Gastroenterol 2020;26:2514-32.

16. Ito T, Sato K, Maekawa H, et al. Elevated levels of serum fatty acid synthase in patients with gastric carcinoma. Oncol Lett 2014;7:616-20.

17. Jiang $M, W u ~ N, X u ~ B$, et al. Fatty acid-induced CD36 expression via O-GlcNAcylation drives gastric cancer metastasis. Theranostics 2019;9:5359-73.

18. Wang J, Wen T, Li Z, et al. CD36 upregulates DEK transcription and promotes cell migration and invasion via GSK-3 $\beta / \beta$-catenin-mediated epithelial-to-mesenchymal transition in gastric cancer. Aging (Albany NY) 2020;13:1883-97.

19. Chang WC, Huang SF, Lee YM, et al. Cholesterol import and steroidogenesis are biosignatures for gastric cancer patient survival. Oncotarget 2017;8:692-704.

20. Ma C, Xie J, Luo C, et al. OxLDL promotes lymphangiogenesis and lymphatic metastasis in gastric cancer by upregulating VEGF-C expression and secretion. Int J Oncol 2019;54:572-84.

21. Wang Q, Feng F, Wang J, et al. Liver X receptor activation reduces gastric cancer cell proliferation by suppressing Wnt signalling via LXR $\beta$ relocalization. J Cell Mol Med 2019;23:789-97.

22. Barrozo RM, Hansen LM, Lam AM, et al. CagY Is an Immune-Sensitive Regulator of the Helicobacter pylori Type IV Secretion System. Gastroenterology 2016;151:1164-1175.e3.

23. Ortiz N, Díaz C. Mevalonate pathway as a novel target for the treatment of metastatic gastric cancer. Oncol Lett 2020;20:320.

24. Sayi A, Kohler E, Hitzler I, et al. The CD4+ T cellmediated IFN-gamma response to Helicobacter infection is essential for clearance and determines gastric cancer risk. J Immunol 2009;182:7085-101.

25. Syu LJ, El-Zaatari M, Eaton KA, et al. Transgenic expression of interferon- $\gamma$ in mouse stomach leads to inflammation, metaplasia, and dysplasia. Am J Pathol 2012;181:2114-25.

26. Kucukazman M, Yavuz B, Sacikara M, et al. The relationship between updated Sydney System score and LDL cholesterol levels in patients infected with Helicobacter pylori. Dig Dis Sci 2009;54:604-7.

27. Morey P, Pfannkuch L, Pang E, et al. Helicobacter pylori Depletes Cholesterol in Gastric Glands to Prevent Interferon Gamma Signaling and Escape the Inflammatory Response. Gastroenterology 2018;154:1391-1404.e9.

28. Lee JS, Kim SH, Lee S, et al. Gastric cancer depends on aldehyde dehydrogenase 3A1 for fatty acid oxidation. Sci Rep 2019;9:16313.

29. Song H, Wang L, Liu HL, et al. Tissue metabolomic fingerprinting reveals metabolic disorders associated with human gastric cancer morbidity. Oncol Rep 2011;26:431-8.

30. Jung J, Jung $\mathrm{Y}$, Bang EJ, et al. Noninvasive diagnosis and evaluation of curative surgery for gastric cancer by using NMR-based metabolomic profiling. Ann Surg Oncol 2014;21 Suppl 4:S736-42.

31. Agustsson T, Rydén M, Hoffstedt J, et al. Mechanism of increased lipolysis in cancer cachexia. Cancer Res 2007;67:5531-7.

32. Luo X, Cheng C, Tan Z, et al. Emerging roles of lipid metabolism in cancer metastasis. Mol Cancer 2017;16:76.

33. Moon YA, Lee JJ, Park SW, et al. The roles of sterol regulatory element-binding proteins in the transactivation of the rat ATP citrate-lyase promoter. J Biol Chem 
2000;275:30280-6.

34. Granchi C. ATP citrate lyase (ACLY) inhibitors: An anticancer strategy at the crossroads of glucose and lipid metabolism. Eur J Med Chem 2018;157:1276-91.

35. Wang MD, Wu H, Fu GB, et al. Acetyl-coenzyme A carboxylase alpha promotion of glucose-mediated fatty acid synthesis enhances survival of hepatocellular carcinoma in mice and patients. Hepatology 2016;63:1272-86.

36. Maan M, Peters JM, Dutta M, et al. Lipid metabolism and lipophagy in cancer. Biochem Biophys Res Commun 2018;504:582-9.

37. Fang W, Cui H, Yu D, et al. Increased expression of phospho-acetyl-CoA carboxylase protein is an independent prognostic factor for human gastric cancer without lymph node metastasis. Med Oncol 2014;31:15.

38. Fhu CW, Ali A. Fatty Acid Synthase: An Emerging Target in Cancer. Molecules 2020;25:3935.

39. Ntambi JM. Regulation of stearoyl-CoA desaturase by polyunsaturated fatty acids and cholesterol. J Lipid Res 1999;40:1549-58.

40. Xiang HG, Hao J, Zhang WJ, et al. Expression of Fatty Acid Synthase Negatively Correlates with PTEN and Predicts Peritoneal Dissemination of Human Gastric Cancer. Asian Pac J Cancer Prev 2015;16:6851-5.

41. Kusakabe T, Nashimoto A, Honma K, et al. Fatty acid synthase is highly expressed in carcinoma, adenoma and in regenerative epithelium and intestinal metaplasia of the stomach. Histopathology 2002;40:71-9.

42. Kim K, Kim HY, Cho HK, et al. The SDF-1alpha/ CXCR4 axis induces the expression of fatty acid synthase via sterol regulatory element-binding protein-1 activation in cancer cells. Carcinogenesis 2010;31:679-86.

43. Menendez JA, Lupu R. Fatty acid synthase and the lipogenic phenotype in cancer pathogenesis. Nat Rev Cancer 2007;7:763-77.

44. Man WC, Miyazaki M, Chu K, et al. Colocalization of SCD1 and DGAT2: implying preference for endogenous monounsaturated fatty acids in triglyceride synthesis. J Lipid Res 2006;47:1928-39.

45. Roongta UV, Pabalan JG, Wang X, et al. Cancer cell dependence on unsaturated fatty acids implicates stearoylCoA desaturase as a target for cancer therapy. Mol Cancer Res 2011;9:1551-61.

46. Xiang F, Wu K, Liu Y, et al. Omental adipocytes enhance the invasiveness of gastric cancer cells by oleic acidinduced activation of the PI3K-Akt signaling pathway. Int J Biochem Cell Biol 2017;84:14-21.

47. Comerford SA, Huang Z, Du X, et al. Acetate dependence of tumors. Cell 2014;159:1591-602.

48. Hur H, Kim YB, Ham IH, et al. Loss of ACSS2 expression predicts poor prognosis in patients with gastric cancer. J Surg Oncol 2015;112:585-91.

49. Metallo CM, Gameiro PA, Bell EL, et al. Reductive glutamine metabolism by IDH1 mediates lipogenesis under hypoxia. Nature 2011;481:380-4.

50. Huang KH, Hsu CC, Fang WL, et al. SIRT3 expression as a biomarker for better prognosis in gastric cancer. World J Surg 2014;38:910-7.

51. Chushi L, Wei W, Kangkang X, et al. HMGCR is upregulated in gastric cancer and promotes the growth and migration of the cancer cells. Gene 2016;587:42-7.

52. Kim ST, Kang JH, Lee J, et al. Simvastatin plus capecitabine-cisplatin versus placebo plus capecitabinecisplatin in patients with previously untreated advanced gastric cancer: a double-blind randomised phase 3 study. Eur J Cancer 2014;50:2822-30.

53. Cheng-Qian Y, Xin-Jing W, Wei-Xinbing, et al. Lovastatin inhibited the growth of gastric cancer cells. Hepatogastroenterology 2014;61:1-4.

54. Carracedo A, Cantley LC, Pandolfi PP. Cancer metabolism: fatty acid oxidation in the limelight. Nat Rev Cancer 2013;13:227-32.

55. Tan $\mathrm{Y}$, Lin K, Zhao Y, et al. Adipocytes fuel gastric cancer omental metastasis via PITPNC1-mediated fatty acid metabolic reprogramming. Theranostics 2018;8:5452-68.

56. Wang Y, Lu JH, Wang F, et al. Inhibition of fatty acid catabolism augments the efficacy of oxaliplatin-based chemotherapy in gastrointestinal cancers. Cancer Lett 2020;473:74-89.

57. Liu Q, Luo Q, Halim A, et al. Targeting lipid metabolism of cancer cells: A promising therapeutic strategy for cancer. Cancer Lett 2017;401:39-45.

58. Rysman E, Brusselmans K, Scheys K, et al. De novo lipogenesis protects cancer cells from free radicals and chemotherapeutics by promoting membrane lipid saturation. Cancer Res 2010;70:8117-26.

59. Nieman KM, Romero IL, Van Houten B, et al. Adipose tissue and adipocytes support tumorigenesis and metastasis. Biochim Biophys Acta 2013;1831:1533-41.

60. Petan T, Jarc E, Jusović M. Lipid Droplets in Cancer: Guardians of Fat in a Stressful World. Molecules 2018;23:1941.

61. Koizume S, Miyagi Y. Lipid Droplets: A Key Cellular Organelle Associated with Cancer Cell Survival under Normoxia and Hypoxia. Int J Mol Sci 2016;17:1430.

62. Matsuoka T, Yashiro M. The Role of PI3K/Akt/ 
mTOR Signaling in Gastric Carcinoma. Cancers (Basel) 2014;6:1441-63.

63. Horton JD, Goldstein JL, Brown MS. SREBPs: activators of the complete program of cholesterol and fatty acid synthesis in the liver. J Clin Invest 2002;109:1125-31.

64. Krycer JR, Sharpe LJ, Luu W, et al. The Akt-SREBP nexus: cell signaling meets lipid metabolism. Trends Endocrinol Metab 2010;21:268-76.

65. Feng WW, Kurokawa M. Lipid metabolic reprogramming as an emerging mechanism of resistance to kinase inhibitors in breast cancer. Cancer Drug Resist 2020. doi: 10.20517/cdr.2019.100.

66. Hirai H, Sootome H, Nakatsuru Y, et al. MK-2206, an allosteric Akt inhibitor, enhances antitumor efficacy by standard chemotherapeutic agents or molecular targeted drugs in vitro and in vivo. Mol Cancer Ther 2010;9:1956-67.

67. Molife LR, Yan L, Vitfell-Rasmussen J, et al. Phase 1 trial of the oral AKT inhibitor MK-2206 plus carboplatin/ paclitaxel, docetaxel, or erlotinib in patients with advanced solid tumors. J Hematol Oncol 2014;7:1.

68. Xing X, Zhang L, Wen X, et al. PP242 suppresses cell proliferation, metastasis, and angiogenesis of gastric cancer through inhibition of the PI3K/AKT/mTOR pathway. Anticancer Drugs 2014;25:1129-40.

69. Li L, Zhang S, Xie D, et al. Dual inhibitor of PI3K and mTOR (NVP-BEZ235) augments the efficacy of fluorouracil on gastric cancer chemotherapy. Onco Targets Ther 2018;11:6111-8.

70. Yan D, Adeshakin AO, Xu M, et al. Lipid Metabolic Pathways Confer the Immunosuppressive Function of Myeloid-Derived Suppressor Cells in Tumor. Front Immunol 2019;10:1399.

71. Zhao R, Wan Q, Wang Y, et al. M1-like TAMs are required for the efficacy of PD-L1/PD-1 blockades in gastric cancer. Oncoimmunology 2020;10:1862520.

72. Gambardella V, Castillo J, Tarazona N, et al. The role of tumor-associated macrophages in gastric cancer development and their potential as a therapeutic target. Cancer Treat Rev 2020;86:102015.

73. Wu H, Han Y, Rodriguez Sillke Y, et al. Lipid dropletdependent fatty acid metabolism controls the immune suppressive phenotype of tumor-associated macrophages. EMBO Mol Med 2019;11:e10698.

74. Zhang Q, Wang H, Mao C, et al. Fatty acid oxidation contributes to IL-1 $\beta$ secretion in M2 macrophages and promotes macrophage-mediated tumor cell migration. Mol Immunol 2018;94:27-35.
75. Corn KC, Windham MA, Rafat M. Lipids in the tumor microenvironment: From cancer progression to treatment. Prog Lipid Res 2020;80:101055.

76. Xu Y, Du X, Turner N, et al. Enhanced acylCoA:cholesterol acyltransferase activity increases cholesterol levels on the lipid droplet surface and impairs adipocyte function. J Biol Chem 2019;294:19306-21.

77. Yang W, Bai Y, Xiong Y, et al. Potentiating the antitumour response of CD8(+) $\mathrm{T}$ cells by modulating cholesterol metabolism. Nature 2016;531:651-5.

78. Zhao Y, Shao Q, Peng G. Exhaustion and senescence: two crucial dysfunctional states of T cells in the tumor microenvironment. Cell Mol Immunol 2020;17:27-35.

79. MacIver NJ, Michalek RD, Rathmell JC. Metabolic regulation of T lymphocytes. Annu Rev Immunol 2013;31:259-83.

80. Yin Z, Bai L, Li W, et al. Targeting T cell metabolism in the tumor microenvironment: an anti-cancer therapeutic strategy. J Exp Clin Cancer Res 2019;38:403.

81. Yuan XL, Chen L, Li MX, et al. Elevated expression of Foxp3 in tumor-infiltrating Treg cells suppresses T-cell proliferation and contributes to gastric cancer progression in a COX-2-dependent manner. Clin Immunol 2010;134:277-88.

82. Kindlund B, Sjöling Å, Yakkala C, et al. CD4+ regulatory $\mathrm{T}$ cells in gastric cancer mucosa are proliferating and express high levels of IL-10 but little TGF- $\beta$. Gastric Cancer 2017;20:116-25.

83. O'Sullivan D, Pearce EL. Fatty acid synthesis tips the TH17-Treg cell balance. Nat Med 2014;20:1235-6.

84. Berod L, Friedrich C, Nandan A, et al. De novo fatty acid synthesis controls the fate between regulatory $\mathrm{T}$ and $\mathrm{T}$ helper 17 cells. Nat Med 2014;20:1327-33.

85. Kimura A, Kishimoto T. IL-6: regulator of Treg/Th17 balance. Eur J Immunol 2010;40:1830-5.

86. Herber DL, Cao W, Nefedova Y, et al. Lipid accumulation and dendritic cell dysfunction in cancer. Nat Med 2010;16:880-6.

87. Fernández LP, Gómez de Cedrón M, Ramírez de Molina A. Alterations of Lipid Metabolism in Cancer: Implications in Prognosis and Treatment. Front Oncol 2020;10:577420.

88. Gabrilovich D. Mechanisms and functional significance of tumour-induced dendritic-cell defects. Nat Rev Immunol 2004;4:941-52.

89. Dong H, Bullock TN. Metabolic influences that regulate dendritic cell function in tumors. Front Immunol 2014;5:24.

90. Choi HS, Ha SY, Kim HM, et al. The prognostic effects of 
tumor infiltrating regulatory $\mathrm{T}$ cells and myeloid derived suppressor cells assessed by multicolor flow cytometry in gastric cancer patients. Oncotarget 2016;7:7940-51.

91. Kim W, Chu TH, Nienhüser H, et al. PD-1 Signaling Promotes Tumor-Infiltrating Myeloid-Derived Suppressor Cells and Gastric Tumorigenesis in Mice. Gastroenterology 2021;160:781-96.

92. Wu H, Weidinger C, Schmidt F, et al. Oleate but not stearate induces the regulatory phenotype of myeloid suppressor cells. Sci Rep 2017;7:7498.

93. Al-Khami AA, Zheng L, Del Valle L, et al. Exogenous lipid uptake induces metabolic and functional reprogramming of tumor-associated myeloid-derived suppressor cells. Oncoimmunology 2017;6:e1344804.

94. Schlaepfer IR, Joshi M. CPT1A-mediated Fat Oxidation, Mechanisms, and Therapeutic Potential. Endocrinology 2020;161:bqz046.

95. Hossain F, Al-Khami AA, Wyczechowska D, et al. Inhibition of Fatty Acid Oxidation Modulates Immunosuppressive Functions of Myeloid-Derived Suppressor Cells and Enhances Cancer Therapies. Cancer Immunol Res 2015;3:1236-47.

Cite this article as: Cui MY, Yi X, Zhu DX, Wu J. Aberrant lipid metabolism reprogramming and immune microenvironment for gastric cancer: a literature review. Transl Cancer Res 2021;10(8):3829-3842. doi: 10.21037/tcr-21-655 